\title{
1 Skin Image Retrieval Using Gabor Wavelet Texture Feature
}

\section{Abstract}

5 OBJECTIVE: Skin imaging plays a key role in many clinical studies. We have used

6 many skin imaging techniques, including the recently developed capacitive contact

7 skin imaging based on fingerprint sensors. The aim of this study is to develop an

8 effective skin image retrieval technique using Gabor wavelet transform, which can

9 be used on different types of skin images, but with a special focus on skin

10 capacitive contact images.

12 METHODS: Content-based image retrieval (CBIR) is a useful technology to retrieve

13 stored images from database by supplying query images. In a typical CBIR, images

14 are retrieved based on colour, shape, and texture, etc. In this paper, texture feature

15 is used for retrieving skin images, and Gabor wavelet transform is used for texture

16 feature description and extraction.

18 RESULTS: The results show that the Gabor wavelet texture features can work

19 efficiently on different types of skin images. Although Gabor wavelet transform is 
20 slower comparing with other image retrieval techniques, such as Principal

21 Component Analysis (PCA) and Grey level co-occurrence matrix (GLCM), Gabor

22 wavelet transform is the best for retrieving skin capacitive contact images and facial

23 images with different orientations. Gabor wavelet transform can also work well on

24 facial images with different expressions and skin cancer/disease images.

CONCLUSION: We have developed an effective skin image retrieval method based

27 on Gabor wavelet transform, that it is useful for retrieving different types of images,

28 namely digital colour face images, digital colour skin cancer and skin disease

29 images, and grayscale skin capacitive contact images. Gabor wavelet transform

30 can also be potentially useful for face recognition (with different orientation and

31 expressions) and skin cancer/disease diagnosis.

33 Keywords

34 Skin imaging, skin capacitive images, content-based image retrieval, Gabor wavelet

35 transform, texture features. 


\section{Introduction}

40 Skin imaging plays a key role in many research areas, such as dermatology, clinical

41 analysis, pharmacology and cosmetic science. In our previous research, we have

42 used many different imaging technologies, including standard digital camera,

43 DermLite Dermoscopy, Proscope HR and the recently developed capacitive contact

44 skin imaging based on fingerprint sensors [1-4]. As the number of skin images

45 soared, image retrieval became more and more important in our research. The aim

46 of this study is to develop an effective content-based image retrieving technique

47 that can work on different types of skin images, but with a particular focus on

48 grayscale capacitive contact skin images, where images are quite similar to each

49 other, and conventional image retrieving techniques normally do not work well.

51 Content-based image retrieval (CBIR), which is one of the popular fundamental

52 research in retrieving accurate useful information, is the set of techniques for

53 retrieving relevant images from an image database on the basis of image features

54 automatically extracted from an image database [5]. In the retrieval phase of a

55 CBIR system, the feature vector for each image in the database is calculated and

56 stored in feature database. After selecting a query image by the user, the matching

57 process that calculates the corresponding feature vector and compares it with all 
58 the feature vectors related to the images in the database is performed. Images

59 having the minimum distance with the query image will be retrieved in a similarity

60 descending order $[6,7]$. In a typical CBIR, images are retrieved based on their visual

61 content such as colour, shape, and texture, etc. [8]. In this paper, texture feature is

62 used for retrieving images.

64 In image processing, texture which generally refers to the structures consisted of

65 large number of texture elements or models similar to each other, it is a key

66 component for human visual perception and plays an important role in

67 image-related applications. Meanwhile, texture features have been researched in

68 the content-based image retrieval, image classification and segmentation.

69 Gray-level co-occurrence matrix (GLCM) [9], Tamura texture feature [10], and

70 Gabor wavelet texture feature [11,12] are the conventional methods used to

71 describe texture feature. Compared with other techniques, Gabor wavelet texture

72 feature is much computationally simpler, and image analysis using Gabor wavelet

73 transform is similar to perception in the human visual system [13]. Gabor wavelet

74 transform has been used in optical character recognition, iris recognition and

75 fingerprint recognition. This paper describes a skin image retrieval technique based

76 on Gabor wavelet texture feature. 


\section{Materials and Methods}

\subsection{Instruments}

80 The main focus of this paper is capacitive contact skin images, but other images are

81 also used to test the performance of the algorithm. All the images used in this study

82 were taken by using standard digital camera and capacitance-based fingerprint

83 sensor, except the skin cancer and skin disease images, which were from Skin

84 Cancer page of About.com [21].

86 Standard digital camera used is SONY DSC--W55 model, which has a 7.2 Mega

Pixels with $3 X$ optical zoom. Capacitance-based fingerprint sensor [2-4] is a novel

88 fringing field capacitive contact image technique that was developed in our research

89 group. It has a matrix of $256 \times 300$ pixels (capacitors) with $50 \mu \mathrm{m}$ spatial resolution

90 per pixel. The total measurement area is $128 \times 15 \mathrm{~mm}^{2}$. The fingerprint sensor

91 basically generates a capacitance image of the skin surface. In each image, each

92 pixel is represented by an 8 bit grayscale value, $0 \sim 255$, the higher grayscale values

93 mean the higher capacitances, and the lower grayscale values mean the lower

94 capacitances. Because in fringing field measurements, capacitance is determined

95 by the dielectric constants of the sample, and water has much higher dielectric 
96 constants than dry skin, therefore, the higher capacitance means the higher water

97 content in skin, vice verse. Apart from water, the sensor is also sensitive to many

98 solvents that have relative high dielectric constant, such as dimethyl sulfoxide

99 (DMSO), ethylene glycol, propylene glycol, propanol, glycerol, and alcohol etc. [2].

100 This makes it a potentially a very useful tool for studying solvent penetrations

101 through membranes or skin, and trans-dermal drug delivery.

\section{$103 \quad 2.2$ Gabor Wavelet Transform}

104 Gabor wavelet can extract the relevant textural feature at different scales and

105 directions in the frequency domain and also has a good joint resolution in both

106 spatial and frequency domain [14]. Gabor wavelet is widely used to extract texture

107 features from the images for image retrieval and has been shown to be very

108 efficient [15-19].

109

110 The typical two dimensional Gabor function can be expressed as the production of

111 Gaussian function and sinusoidal function [19,20]:

112

$$
g(x, y)=\frac{\square}{\square \pi \sigma_{x} \sigma_{y}} e^{-\frac{1}{2}\left(\frac{x^{2}}{\sigma_{x}^{2}}+\frac{y^{2}}{\sigma_{y}^{2}}\right)} \square\left(e^{2 \pi j f x}\right)
$$


115 Where $\sigma_{x}$ and $\sigma_{y}$ are the Gaussian variance, which describe the spreads of the

116 Gaussian function, $\boldsymbol{j}$ is the imaginary unit of complex number, $\boldsymbol{f}$ is the frequency of

117 the sinusoidal function. Using Eq. 1 as the mother function, we can generate a set

118 of child functions, called Gabor wavelets.

$$
\begin{aligned}
& g_{m n}(x, y)=a^{-m} g\left(x^{\prime}, y^{\prime}\right) \quad a>1, m, n=\text { integer } \\
& x^{\prime}=x \cos \theta+y \sin \theta \\
& y^{\prime}=-x \sin \theta+y \cos \theta
\end{aligned}
$$

122

123 where $\theta=n \pi / K, \mathrm{n}=0,1, \ldots, \mathrm{K}-1$, and $K$ is the total number of the directions

124 which specifies the orientation of a Gabor function; $m=0,1, \ldots, S-1$, and $S$ is the 125 number of scales which specifies the amplitude of a Gabor function. If we use

$126\left(U_{l}, U_{h}\right)$ to denote the lower and upper centre frequency of the sinusoidal function, 127 we have

128

$$
\begin{aligned}
& a=\frac{\square U_{h}{ }^{1}}{\square-1} \\
& \square=U_{l} \square \\
& f=U_{h}
\end{aligned}
$$


131 In this paper, the total number of directions $(K)$ and scales $(S)$ and have chosen to

132 be $K=6$ and $S=4$, respectively, which is resulting $4 \times 6=24$ Gabor wavelet

133 filters to filter the images. $U_{l}$ and $U_{h}$ used are 0.05 and 0.4 , respectively.

134

135 Figure1 (A) shows typical profiles of Gaussian function, sinusoidal function and the

136 corresponding wavelet function; (B) shows the Gabor wavelet profiles at 6 different

137 directions and 4 different scales.

138

(Figure 1 goes in here)

140

141 Gabor wavelet transform can be considered as a wavelet transform whose mother

142 wavelet is Gabor function. For a given image $I(x, y)$ with $M \times N$ pixels, its Gabor

143 wavelet transform is defined as follows:

144

145

$$
W_{n m}(x, y)=\int I\left(x_{1}, y_{1}\right) g_{n m} *\left(x-x_{1}, y-y_{1}\right) d_{x_{1}} d_{y_{1}}
$$

146

147 where * represents the complex conjugate. 


\subsection{Experimental Procedures}

151 A image database that includes 56 images in JPEG format was setup. Figure 2

152 shows some sample images from the database, which contains three different

153 types of images: human faces, skin cancer and skin disease images [21], and skin

154 grayscale capacitive contact images.

155

156

(Figure 2 goes in here)

157

158 A software programme has been developed to search above image database using

159 a query image, based the Gabor wavelet transform. A Graphic User Interface (GUI)

160 was also developed to simplify the operations. Following are the steps of the

161 programme:

162 1. Convert all the colour images into gray images.

1632 2. Perform the Gabor wavelet transform on all images.

$1643 . \quad$ Calculate the mean and standard deviation as a texture feature.

1654 4. Compare the texture feature of the query image with that of images in the 166 database, in order to find the best match results. 


\section{Results and Discussions}

\section{$170 \quad 3.1$ Image query results}

171 Figure 3 shows the image retrieval results using Gabor texture features. In all the

172 four retrieval results shown, the best three retrieved images are shown for

173 illustration. The retrieved images are ranked in descending order according to the

174 similarity of their Gabor texture features to those of the query image, i.e. the most

175 similar, the second similar, and the third similar images. In this study, for simplicity

176 reasons, the query images are also from the database, and therefore the most

177 similar result should always be the image itself.

178

180

181 In Figure 3(A), the query image is a grey capacitive image of finger skin, where

182 finger friction ridges are clearly visible. The black spots are the areas that water is

183 actively coming out of skin. As it can be seen, most of the best 3 retrieved images

184 are all similar finger grey capacitive images captured from Fingerprint sensor.

185 Figure 3(B) shows the retrieval results for a human face query image. Human face

186 images in the database are with two different expressions - smile and non-smile.

187 The most similar image is the same of query image. The second most similar image 
188 is the same person without smile and the third image is that another person without

189 smile. It shows that this method has certain reference value for human face

190 recognition. It might be even also useful for facial expression recognition. Figure

$1913(C)$ and $3(D)$ are the output of skin cancer/disease retrieval results. The query

192 image in Figure 3(C) is a skin cancer image of melanoma. From the illustration, the

193 three of the most similar images are all skin cancer images. The query image in

194 Figure 3(D) is a skin disease image of leucoderma. From the results, the three of

195 the most similar images are all leucoderma images. The results show that Gabor

196 wavelet transform could also be potentially used for skin disease diagnostics. Users

197 could take a skin image, and search the skin disease database, and find out what

198 possible type of skin disease it might resemble, and seek doctors for early

199 diagnoses.

200

$201 \quad 3.2$ The effects of scales (S) and directions (K)

202 The values of scales (S) and directions (K) in Gabor wavelet transform not only

203 affect the accuracy of retrieval, they will also affect the computational time of

204 program. Figure $4(\mathrm{~A})$ and $4(\mathrm{~B})$ show typical retrieval errors when SxK $=2 \times 2$ and

205 Figure 4(C) shows the relationships between computational time and the production

206 of scales and directions (SxK). If any of the best three results is not the right type of 
207 images, such as Figure 4(A) or 4(B), we classify it as a retrieval error. Figure 4(C)

208 shows the relationships between retrieval errors and SxK. Generally speaking, the

209 higher the values of SxK, the lower the retrieval errors, but the longer the

210 computational time; the lower the values of SxK, the shorter the computational time,

211 but the higher the retrieval errors. The key is to find the optimum value of SxK that

212 has highest accuracy but lowest possible computational time. From this study, it is

213 found that setting scales and directions to 4 and 6 is a reasonable choice.

(Figure 4 goes in here)

\section{$216 \quad 3.3$ The effects of $U_{l}$ and $U_{h}$}

217 Although the values of lower and higher centre frequency $U_{l}$ and $U_{h}$ do not affect

218 the computational time, they do effect the retrieval accuracy. In general, the values

219 of $U_{l}$ and $U_{h}$ are set to 0.05 and 0.4 because the lowest frequency of image is 0

220 and the highest frequency of image calculated from Nyquist sampling theorem is

221 0.5. According to the visual characteristics of the human eye, the frequency range

222 from 0.05 to 0.4 can be completely reflect people's perception of texture features

223 [22]. However, the retrieval results using these standard values shown in Figure 5

224 (A) are unsatisfactory because the query image in Figure $5(\mathrm{~A})$ is the neck grey

225 capacitive image, but the third most similar image is the face grey capacitive image. 
226 By changing the value of $U_{l}$ and $U_{h}$ to 0.2 and 0.3 and the results shown in Figure

2275 (B) become much better. From Figure 5, it can be concluded that the values of $U_{l}$

228 and $U_{h}$ might need to be adjusted differently to different type of images in order to

229 have better retrieval accuracy.

(Figure 5 goes in here)

234 3.4 The performance of Gabor Wavelet Transform against other algorithms

235 In order to understand the performance of Gabor wavelet transform, we did an 236 image retrieval comparison study, compare Gabor wavelet transform against other

237 two algorithms: Principal Component Analysis (PCA) and Grey level co-occurrence 238 matrix (GLCM).

240 Table 1 shows the computation time of different algorithms. The results show that

241 GLCM is the fastest for calculating the feature vectors of all the images in the 242 database, whilst Gabor wavelet transform is the slowest. PCA is the fastest for 243 retrieving images, and Gabor wavelet transform is again the slowest. 
245 Table 1 The computation time used of each algorithm.

\begin{tabular}{|l|l|l|l|}
\hline & PCA & GLCM & Gabor Wavelet \\
\hline Time 1 & Average & Low & High \\
\hline Time 2 & Low & Average & High \\
\hline
\end{tabular}

246 Time 1: the time for calculating the feature vectors of all the images in the database.

247 Time 2: the time for retrieving the query image.

249 Table 2 The retrieval success rates of each algorithm.

\begin{tabular}{|l|l|l|l|}
\hline & PCA & GLCM & Gabor Wavelet \\
\hline Image type 1 & Best & Worst & Average \\
\hline Image type 2 & Average & Worst & Best \\
\hline Image type 3 & Worst & Average & Best \\
\hline Image type 4 & Worst & Best & Average \\
\hline
\end{tabular}

250 Image type 1: human face images with different expressions.

251 Image type 2: human face images with different orientations.

252 Image type 3: grayscale capacitive skin images of different parts of human body.

253 Image type 4: skin diseases and skin cancer images. 
255 Table 2 shows the successful retrieval rates of three different algorithms on different

256 type of skin images. Gabor wavelet transform is the best for retrieving capacitive

257 skin images and facial images with different orientations, GLCM is the best for

258 retrieving skin cancer / disease images, PCA is the best for retrieving facial images

259 with different expressions. The results also show that Gabor wavelet transform

260 works reasonably well for human faces with different expressions and skin cancer

261 and disease images.

2634 Conclusions and Future Works

264 We have developed an effective skin image retrieval method based on Gabor

265 wavelet transform. Experimental results show that it is useful for retrieving different

266 types of images, namely digital colour face images, digital colour skin cancer and

267 skin disease images, and particularly suitable grayscale skin capacitive images.

268 The results also suggest that using the Gabor wavelets to extract texture features

269 could be useful for recognizing human face with different orientations and different

270 facial expressions, as well as for skin cancers and diseases diagnostics etc. In

271 Gabor wavelet transform, the values of scales, directions, lower and higher centre

272 frequency, might need to be adjusted differently according to different types of 
273 images, in order to achieve a better retrieval results. For future work, we will

274 investigate to use another visual content such as colour and shape to retrieve 275 images.

276

277 Acknowledgements

278 We thank London South Bank University for the finance support of this project. 


\section{References}

281 1. Singh, H., Development of a Measurement Instrument Using Capacitance 282 Sensors Techniques to Image and Measure the Skin Surface Hydration, PhD 283 Thesis, London South Bank University, November 2010.

284 2. Singh, H., Xiao, P., Berg, E.P., and Imhof, R.E., Skin Capacitance Imaging for 285 Surface Profiles and Dynamic Water Concentration Measurements, ISBS 286 Conference, Seoul, Korea, May 7-10, 2008.

3. Ou, X., Pan, W., Xiao, P., In vivo skin capacitive imaging analysis by using grey 288 level co-occurrence matrix (GLCM), International Journal of Pharmaceutics, Available online 2 November 2013, ISSN 0378-5173, $290 \quad$ http://dx.doi.org/10.1016/j.ijpharm.2013.10.024.

291 4. Xiao P., Ou X., Ciortea L.I., Berg E.P., Imhof R.E., In Vivo Skin Solvent 292 Penetration Measurements Using Opto-thermal Radiometry and Fingerprint 293 Sensor. Int J Thermophys. 33, 2012, 1787-1794.

294 5. Wang J.Z., Integrated Region-Based Image Retrieval. Boston:Kluwer Academic $295 \quad 2001,1-5$.

296 6. Rafiee, G., A review of content-based image retrieval. International Symposium 297 on Communication Systems Networks and Digital Signal Processing (CSNDSP), $298 \quad 2010,7,775-779$. 
299 7. Smeulders, A.W.M., Worring, M., Santini, S., Gupta, A., Jain, R., Content-Based

300 Image Retrieval at the End of the Early Years. IEEE TRANSACTIONS ON PATTERN ANALYSIS AND MACHINE INTELLIGENCE. 22, 2000, 1349-1380.

8. Gong, Y., Zhang, H., Chuant, H. and Skauuchi. M., An image database system with contents capturing and fast image indexing abilities. Proceedings of IEEE International Conferences on Multimedia Computing and Systems, Boston,Massachusetts, USA. 1994,5, 121-130.

9. Haralick, R.M., Shanmugam, K., and Dinstein. I., Texture features for image classification. IEEETrans. Syst. Man Cybern. SMC(8), 1973,11, 610-621.

10. Hayes, K.C., Shah, Jr. A.N., and Rosenfeld, A., Texture coarseness: further experiments. IEEETrans SMC-4, 467-472.

11. Yang, J., Liu, L., Jiang T., and Fan, Y., A modified Gabor filter design method

311 for fingerprint image enhancement. Pattern Recognition Letters. 24(12), 2003, $312 \quad 1805-1817$.

313 12. Daugman, J.G., Uncertainty relation for resolution in space, spatial frequency, 314 and orientation optimized by twodimensional visual cortical filters. Journal of The 315 Optical Society of America. 2(7), 1985, 1160-1169. 
316 13. Kruizinga, P., Petkov, N., and Grigorescu, S.E., Comparison of texture features

317 based on Gabor filters. Proceedings of the 10th International Conference on

318 Image Analysis and Processing. September 27-29, 1999, pp.142-147.

319 14. Smith, J.R., "Integrated Spatial and Feature Image System: Retrieval, Analysis 320 and Compression". Ph.D thesis, Columbia University, 1997.

321 15. Deng, Y., "A Region Representation for Image and Video Retrieval". Ph.D 322 thesis, University of California, Santa Barbara, 1999.

323 16. Dimai, A., Rotation Invariant Texture Description using General Moment 324 Invariants and Gabor Filters. Proc. Of the 11th Scandinavian Conf. on Image $325 \quad$ Analysis. I, 1999, 6, 391-398.

326 17. Liu, Z, An Improved Face Recognition Method Based on Gabor Wavelet 327 Transform and SVM. Fifth International Symposium on Computational 328 Intelligence and Design (ISCID), 2012,10, 378 - 381.

329 18. Fischer, S., Minimum entropy transform using Gabor wavelets for image 330 compression. 11th International Conference on Image Analysis and Processing 331 and Proceedings, 2001,9,428 - 433.

332 19. Manjunath, B.S., and Ma, W.Y., Texture features for browsing and retrieval of 333 image data. IEEE Trans. Pattern Anal. Machine Intell. 18,1996,8, 837-842. 
334 20. Skin cancer and skin diseases images,

335 http://skincancer.about.com/od/symptoms/ss/mole_all.htm

336 21. Anne H., Solberg S., Jain A.K. Texture fusion and feature selection applied to 337 SAR imagety [J]. IEEE transaction on Geoscience and Remote sensing. 35(2), $338 \quad 1997,475-478$.

339 340 


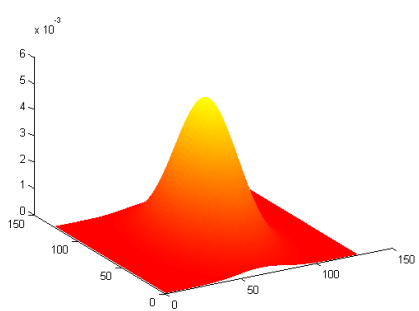

342

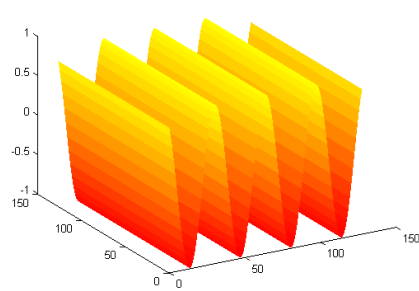

Sinusoidal function

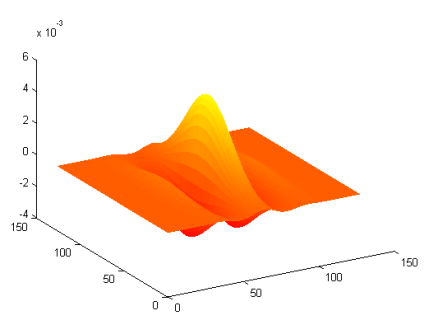

Gabor function

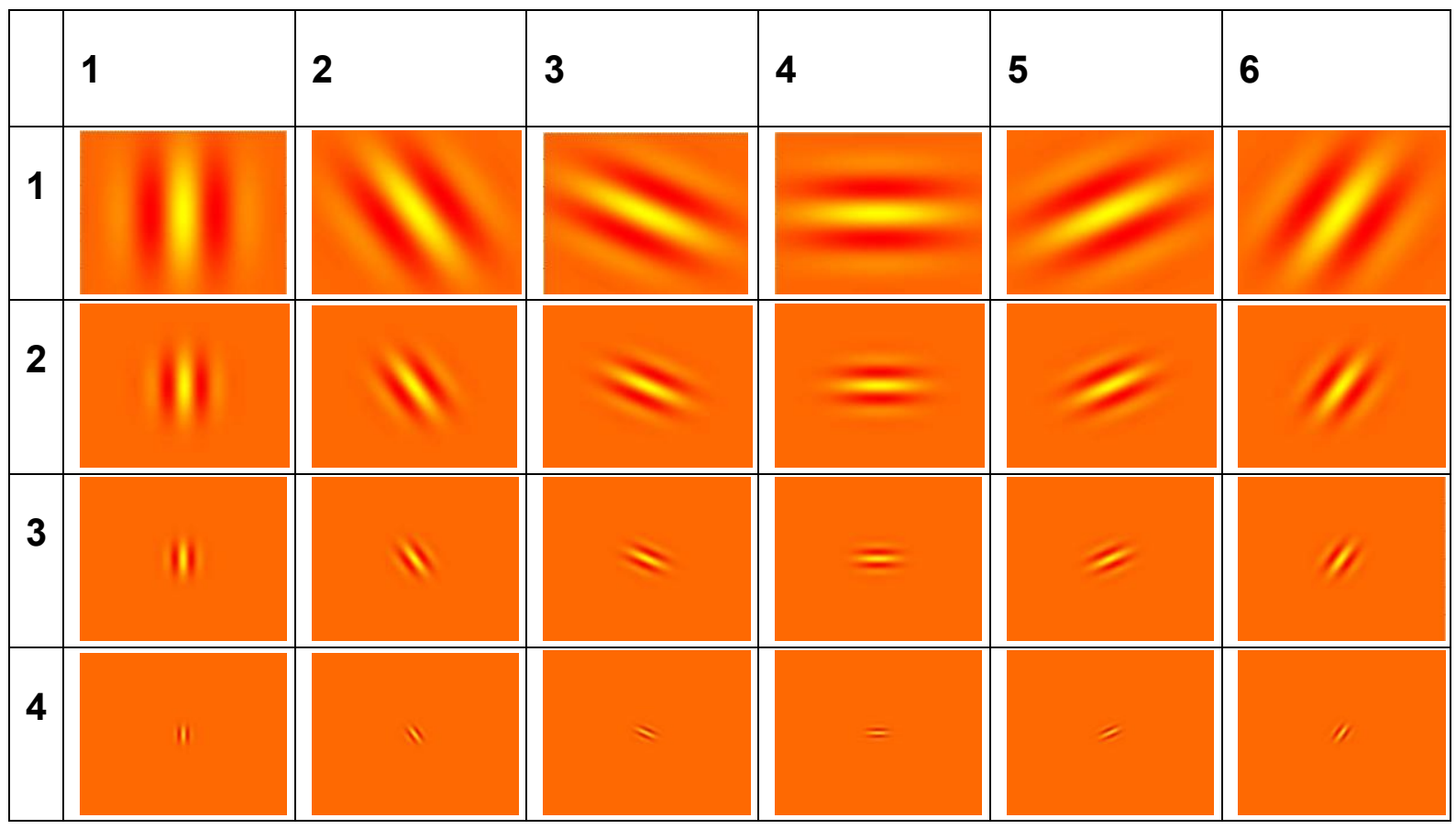

344 (B) Top view of Gabor wavelet profiles at 6 different directions (columns) and 4 345 scales (rows).

347 Figure 1. Gabor wavelet profiles with at different directions and scales. 

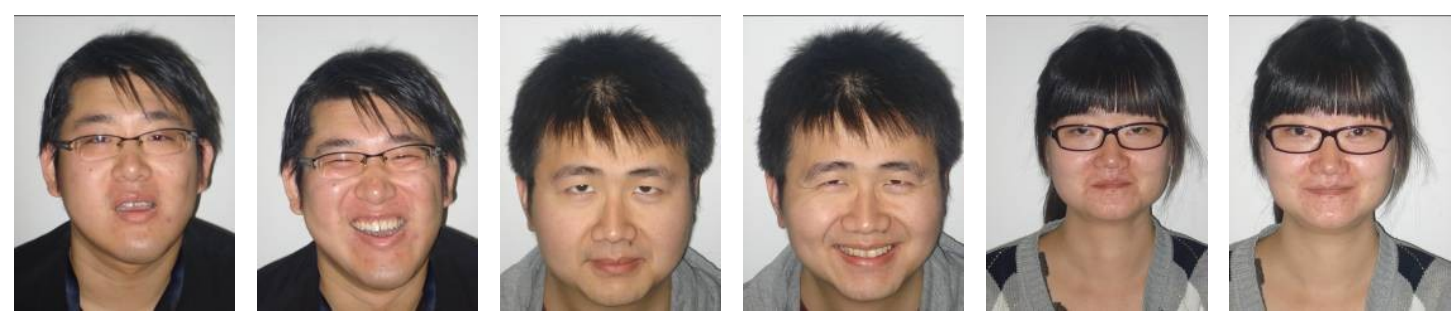

\section{Faces}
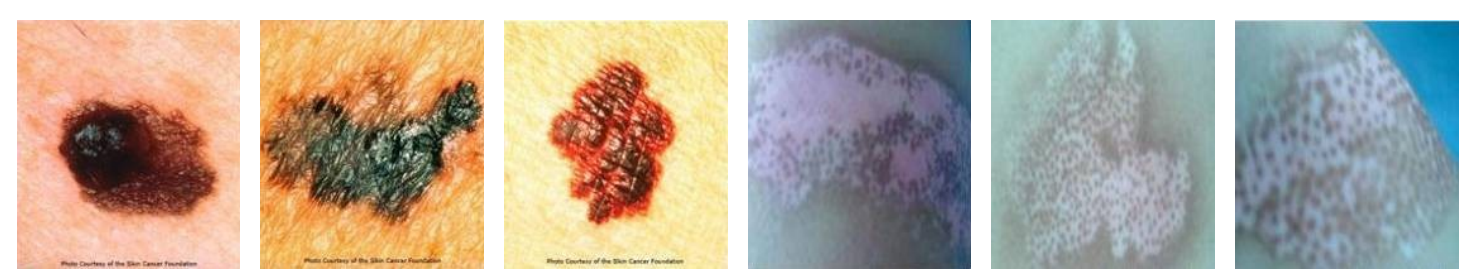

353

Skin cancers

Skin diseases

354
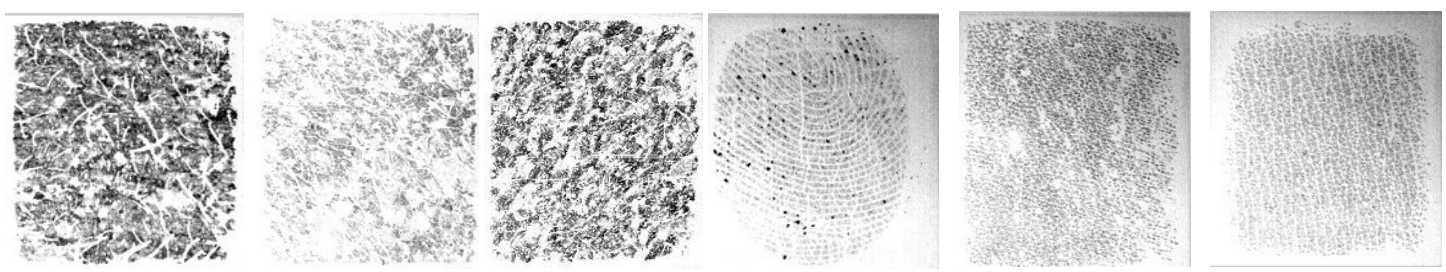

Capacitive skin images

357 Figure 2. Sample images from the database. 
(A)

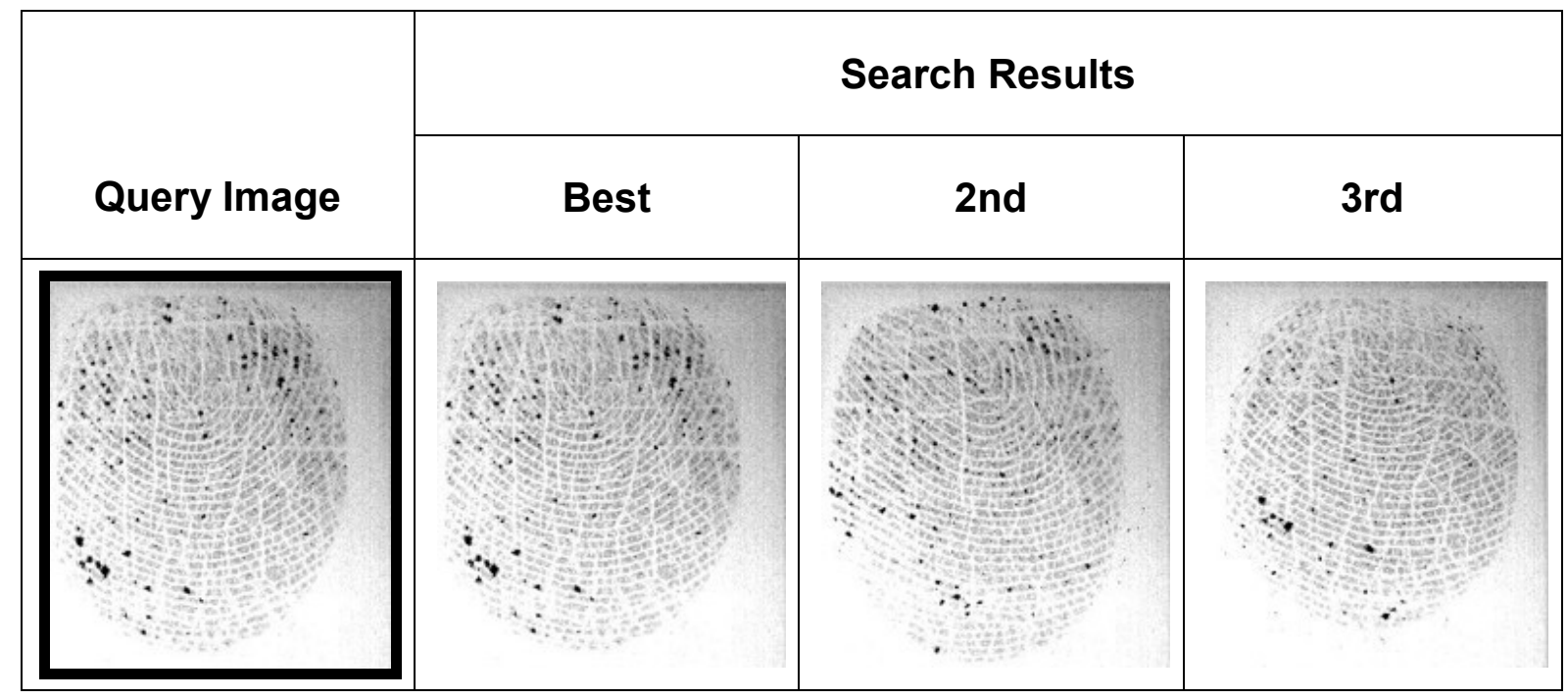
(B)

\begin{tabular}{|c|c|c|c|}
\hline \multirow{2}{*}{ Query Image } & \multicolumn{3}{|c|}{ Search Results } \\
\cline { 2 - 4 } & Best & 2nd & 3rd \\
\hline \multirow{2}{*}{ Q } & \multicolumn{2}{|c|}{ 2nd } \\
\hline
\end{tabular}


(C)

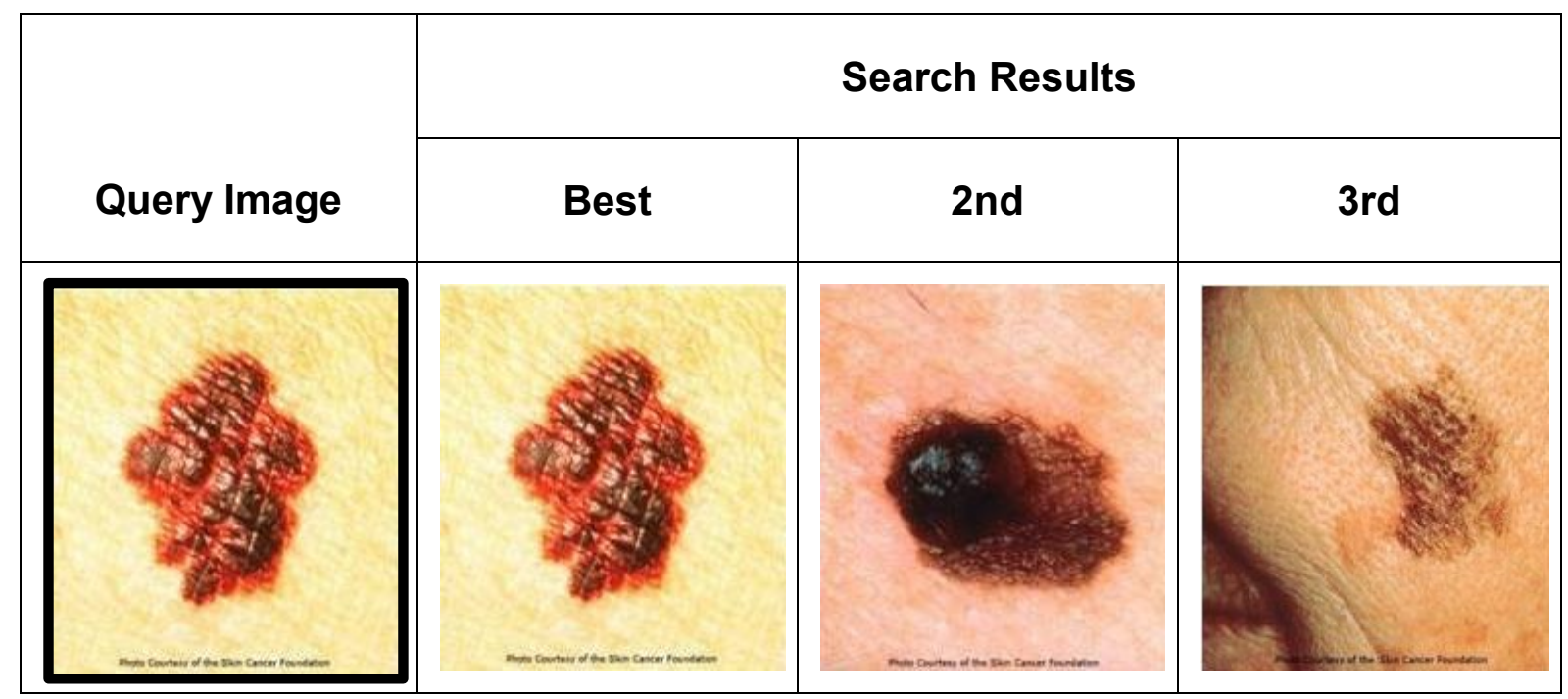

(D)

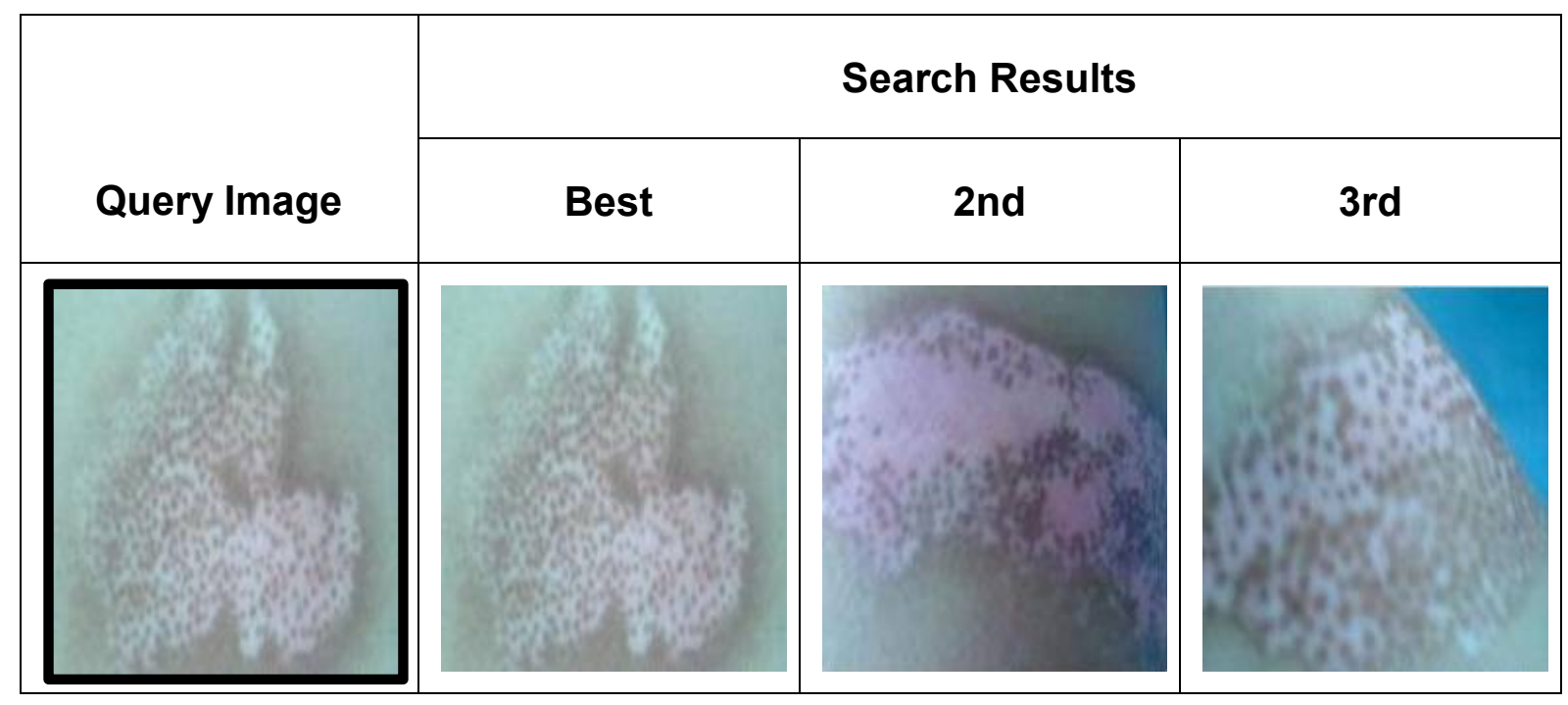

371 Figure 3. Image retrieval results using Gabor texture features. 


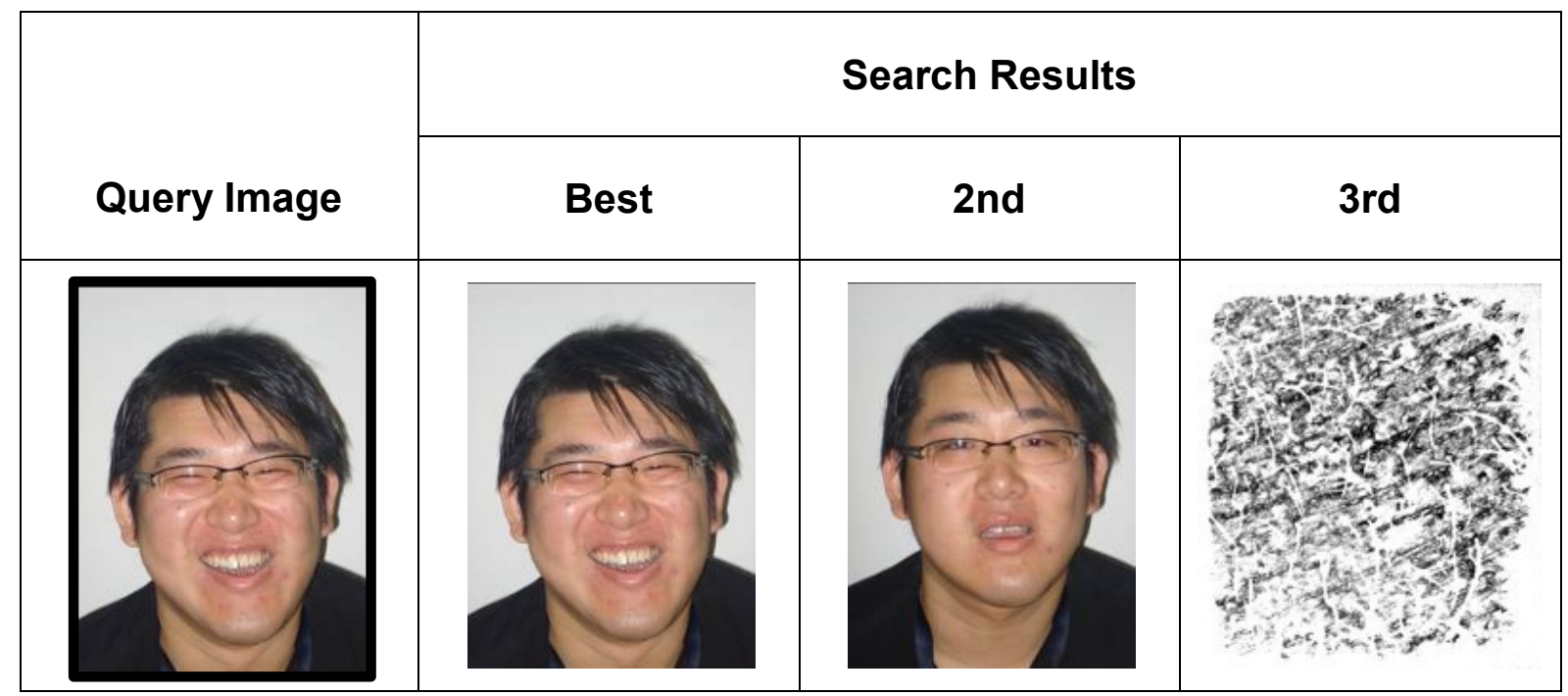

(B)

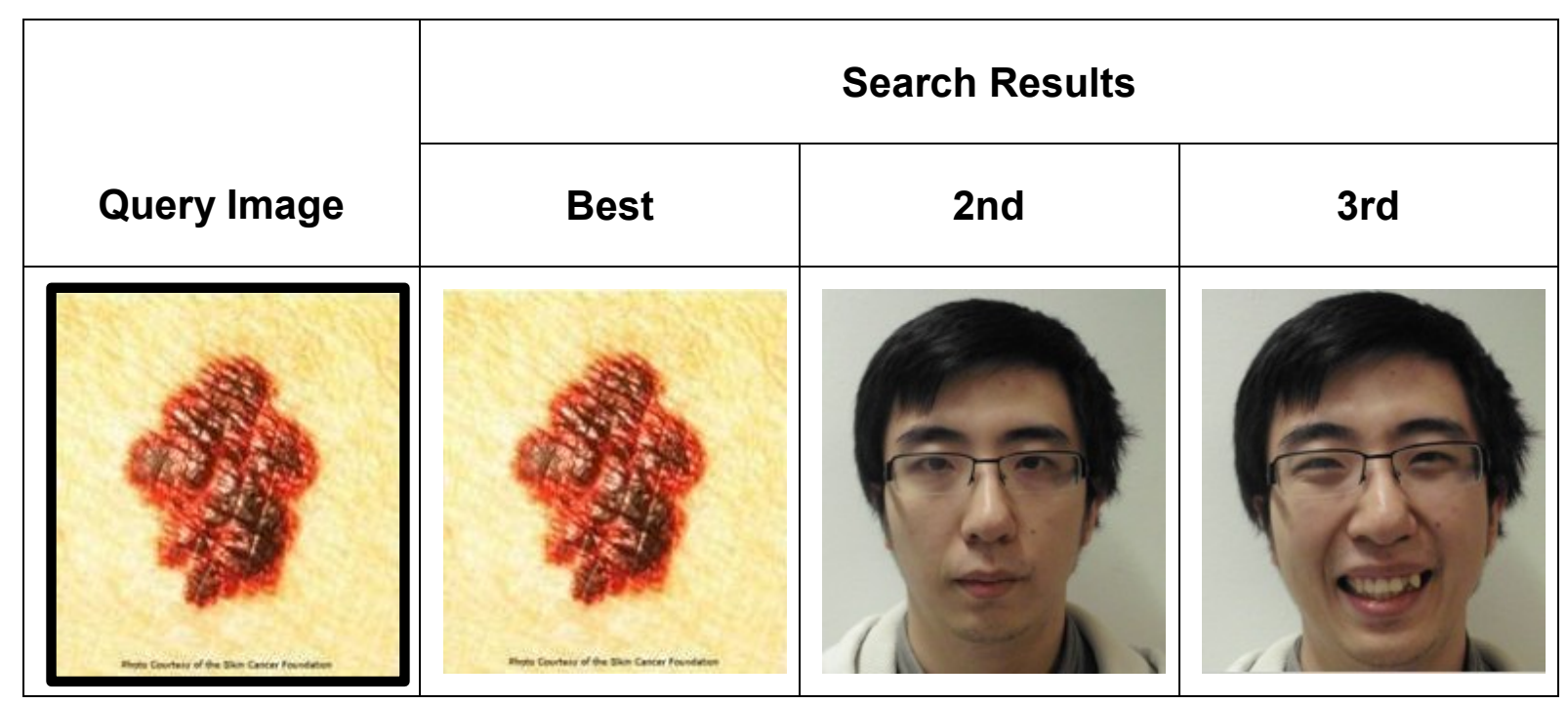




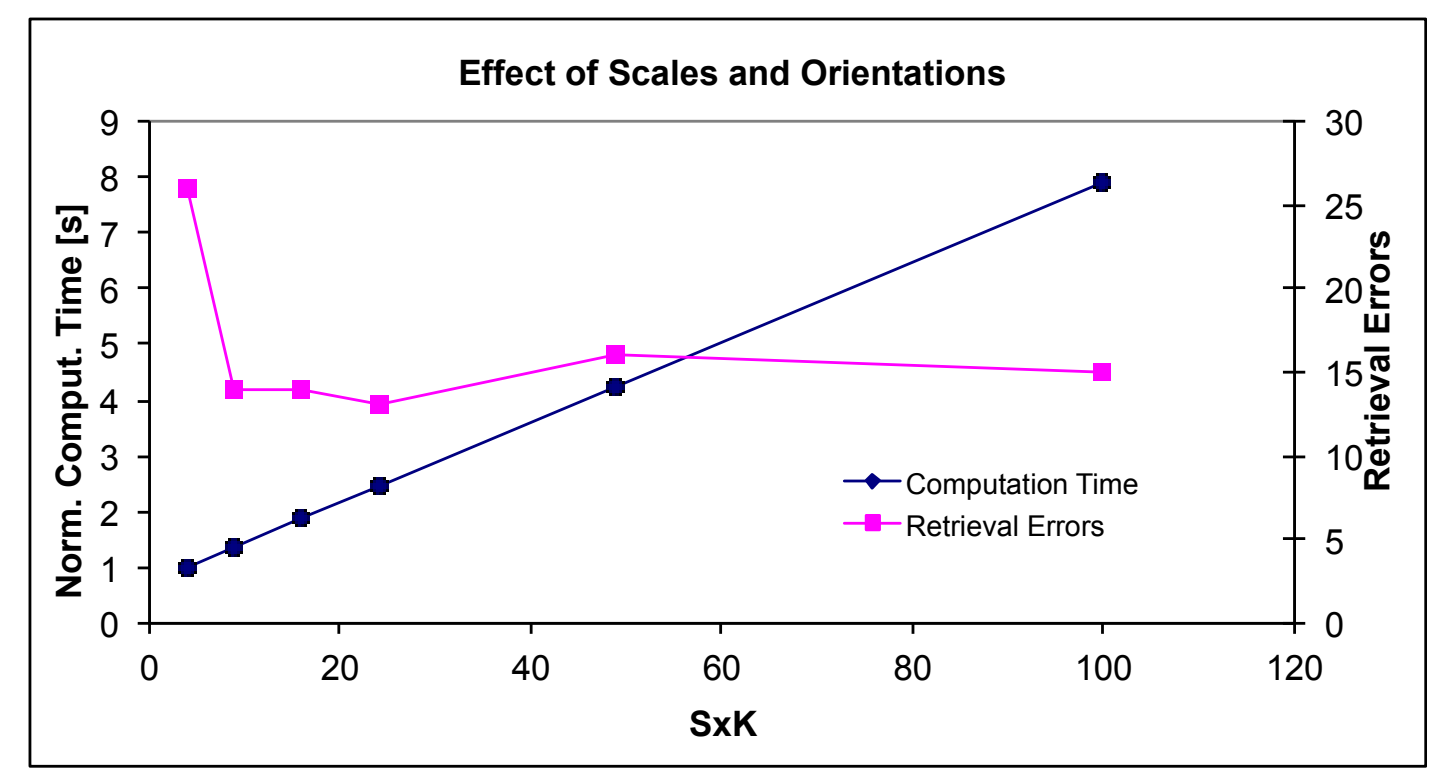

385 Figure 4. (A) and $(B)$ are typical image retrieval errors when SxK $=2 \times 2,(C)$ is 386 computational times and retrieval errors against the production of scales and 387 directions SxK. 
(A)

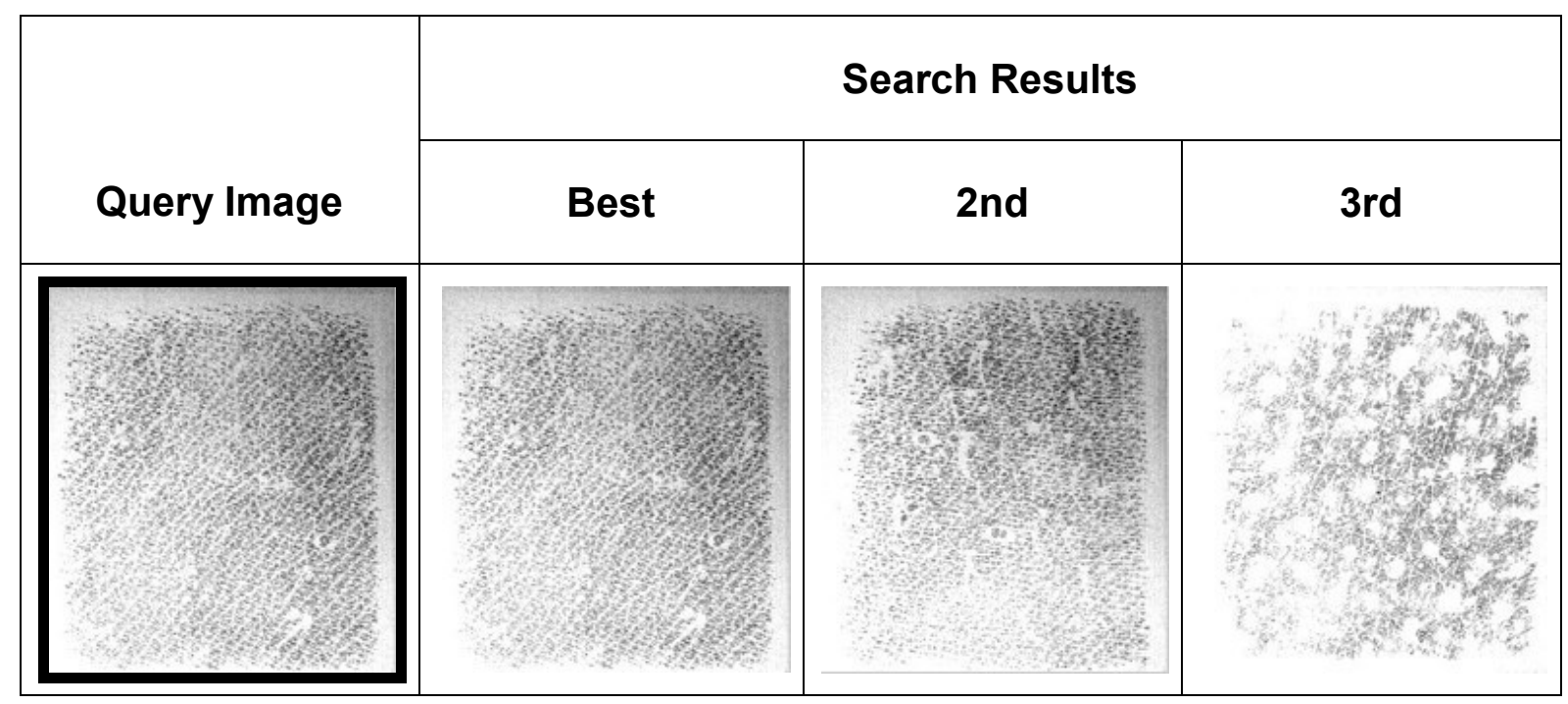

(B)

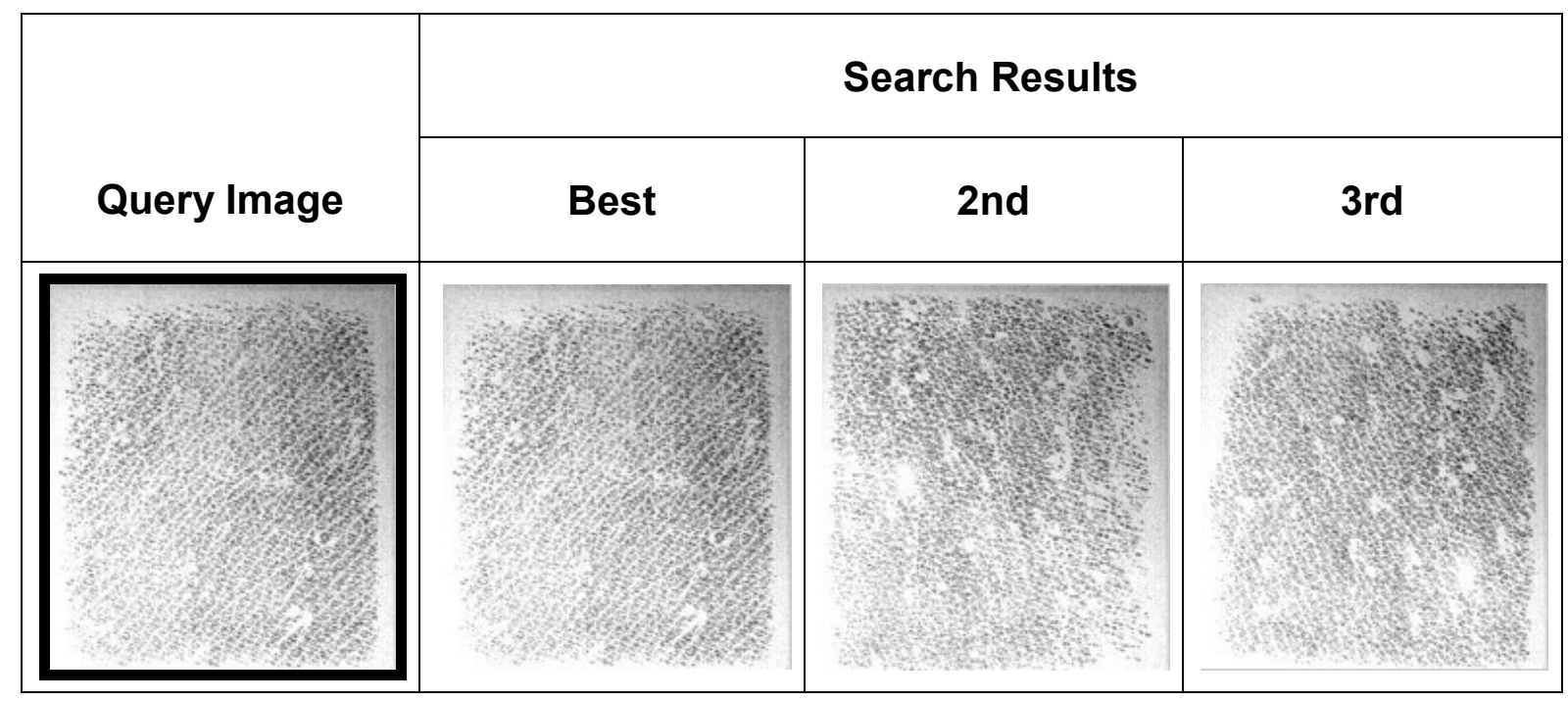

394 Figure 5. Image retrieval results using Gabor texture features when (A) $U_{l}=0.05$

395 and $U_{h}=0.4$ and $(\mathrm{B}) U_{l}=0.2$ and $U_{h}=0.3$. 